\title{
PROSPECCIÓN DE LA ESPECIE Commelina benghalensis L. (Commelinaceae) EN LA REGIÓN CENTRAL Y SUR DE NICARAGUA Y ANÁLISIS DE RIESGO DE SU INTRODUCCIÓN A TRAVÉS DE SEMILLA DE PASTOS
}

\author{
EXPLORATION OF THE SPECIES Commelina benghalensis L. (Commelinaceae) IN THE CENTRAL AND SOUTH \\ REGIONS OF NICARAGUA AND RISK ANALYSIS OF ITS INTRODUCTION THROUGH PASTURE SEED
}

\section{Freddy Alemán}

Universidad Nacional Agraria, apartado 453. E-mail: freddy@una.edu.ni

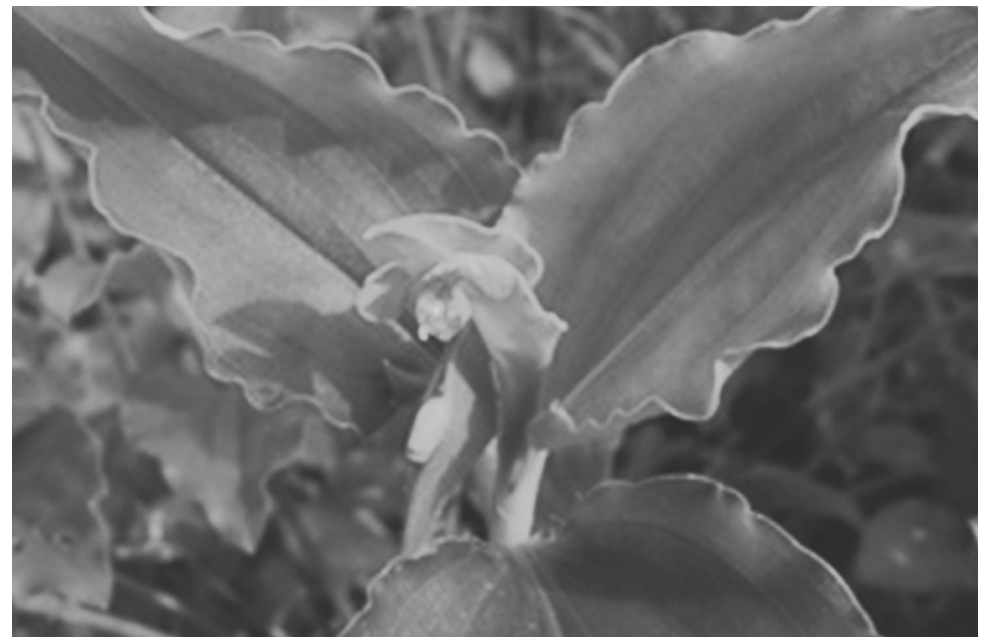

\section{RESUMEN}

La especie Commelina bengalensis L. es una maleza de alto riesgo y reconocida agresividad, la cual, conforme legislación vigente en Nicaragua, tiene restringida la introducción al país. En vista de lo anterior, se llevó a cabo un estudio con el propósito de determinar la presencia-ausencia de dicha especie en la región sur-central y sur de Nicaragua, y en caso de ausencia, realizar un análisis de riesgo de introducción de la especie a través de la importación de semilla de pastos. El estudio se llevó a cabo en los departamentos de Boaco, Chontales, Rivas y el municipio de Nueva Guinea. El estudio es de naturaleza no experimental, de corte trasversal. En un periodo de 15 días, entre el 15 de marzo y el 30 de marzo del año 2012 se llevaron a cabo muestreos sistemáticos en pastizales de 14 fincas ganaderas en las regiones mencionadas. La información obtenida fue presencia o ausencia de la especie en mención, e información referente a otras especies del genero Commelina, con el propósito de corroborar o descartar la presencia de la especie. Posteriormente, en vista de la ausencia de la especie en los campos muestreados, en trabajo de gabinete se realizó una revisión documental con el propósito de hacer una evaluación del riesgo de introducción de la especie. Los resultados muestran ausencia de la especie. Durante el estudio fue posible determinar la presencia de las especies $C$. diffusa y $C$. erecta, las cuales muestras similitudes con $C$. benghalensis, sin embargo, particularidades morfológicas de esta última permiten descartar que se trate de dicha especie. Para evitar la introducción y establecimiento de C. benghalensis se debe exigir que las semillas de pastos importadas provengan de sitios libres de la especie, y donde se realice un manejo fitosanitario que garantice que el producto esté libre de semilla de la especie, realizar programa de verificación en

\section{ABSTRACT}

Commelina bengalensis L. is recognized as high-risk and aggressive weed specie which under Nicaraguan current legislation has restricted the introduction to the country. In view of this, a study was conducted in order to determine the presence or absence of this species in the south-central and southern part of Nicaragua, and in case of absence, to perform a risk analysis of introduction of the species through pasture seed. The study was conducted in the departments of Boaco, Chontales, Rivas and the municipality of Nueva Guinea. The study is not experimental, cross cutting in nature. In a period of 15 days, from 15 March to 30 March 2012, 14 grassland farms were sampled systematically sampled in the regions mentioned. The information obtained was presence or absence of the specie, and information regarding other species of the genus Commelina, in order to confirm or rule out the presence of the $C$. benghalensis. Subsequently, in view of the absence of the specie, a literature review was conducted in order to evaluate the risk of introduction it. Results show absence of the specie. During the study it was possible to determine the presence of the species $C$. diffusa and $C$. erecta with similarities with $C$. benghalensis, however, morphological peculiarities of the latter allow concerned discard of such specie. To prevent the introduction and establishment of C. benghalensis it is require that imported grass seeds come from sites free of the specie, to be sure that phytosanitary management is performed to ensure that the pasture seeds are free from seeds of the specie, the creation of a verification program at the origin country to determine the origin of the seeds and fulfill the requirements established in a regular seed production programs. Likewise, to request international phytosanitary certificate stating 
los sitios de origen para determinar la procedencia de las semillas, así como cumplimiento de los requisitos que se establecen en programas de producción de semillas, exigir certificado fitosanitario internacional que indique que el producto proviene de áreas libres la maleza y localmente realizar inspección, muestreo y análisis de laboratorio para determinar la ausencia o presencia de la maleza.

Palabras clave: Malezas, arvenses, herbología, semilla contaminada, importación de semilla, cuarentena.

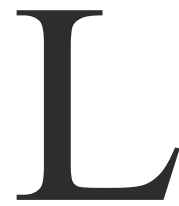

a especie Commelina benghalensis L. pertenece a la familia Commelinaceae, ampliamente distribuida en regiones tropicales y subtropicales de Asia, África, América del Norte y del sur (Cristóbal et al., 2008). Es una especie de interés agronómico por tratarse de una maleza de difícil erradicación, por su condición de producir dos clases de frutos, unos que maduran al aire y otros que lo hacen bajo tierra, y por su marcada resistencia a los herbicidas (Correia, 2008).

Esta especie es considerada una de las malezas más agresivas en diversas partes del mundo. Su control agronómico es dificultoso causando pérdidas económicas importantes en todo el mundo (Grabiele et al., 2009). Es nativa de de África y Asia tropical, aunque ha sido introducida en casi todo el globo. En América se la registra creciendo en campo abierto, borde de bosques y áreas cultivadas en Antillas, Argentina, Brasil, Bolivia, Guyana Francesa, México, Paraguay y sur de USA.

En los últimos años, en Nicaragua, la importación de semilla de pastos se ha visto restringida debido a las amenazas que representa la introducción de la maleza Commelina bengalensis L. Semillas de esta especie han sido determinadas en cargamentos de semillas de pastos importados de Brasil $^{1}$, país donde la maleza es reportada compitiendo con diversos cultivos. La presencia de material reproductivo de la especie ha obligado al rechazo de embarques de semilla de pastos debido a que $C$. benghalensis es sujeta de cuarentena en Nicaragua. Esta situación ha creado incertidumbre, $\mathrm{y}$ ha vislumbrado la posibilidad de que la especie ya se encuentre establecida en nuestros campos, específicamente en pastizales de la región central y sur de Nicaragua, en las cuales prevalece la actividad ganadera.

La presencia de esta especie en campos cultivados o abiertos, no ha sido reportada en Nicaragua, razón por la cual se han enunciado medidas para evitar la introducción al país. Conforme la legislación vigente en Nicaragua, la Norma Técnica Obligatoria Nicaragüense 11 005-02 para

\footnotetext{
${ }^{1}$ Vallecillo, P. 2012. Detección de semillas de C. benghalensis en semillas de pasto. Managua. Dirección General de Sanidad Agropecuaria (DGSA), Managua, Nicaragua. Comunicación personal.
}

that the product comes from the weed-free areas, and locally perform inspection sampling and laboratory analysis to determine the absence or presence of the weed.

Keywords: Weeds, herbology, contaminated seed, seed importation, quarantine.

la importación de productos y subproductos de origen vegetal y organismos vivos de uso agrícola. (MAGFOR, 2002) establece normas y reglas para la producción y comercialización de semillas de especies forrajeras de clima tropical. Las semillas de la especie $C$. benghalensis, se constituyen una de las especies reconocidas como maleza agresiva de alto riesgo y la presencia en semillas comerciales es limitada, conforme normas y reglas establecidas.

El alto riesgo que constituye la presencia de esta especie en las áreas cultivas de nuestro país obliga a las autoridades fitosanitarias a decretar medidas tendientes a evitar su introducción y establecimiento. Por ser una especie en cuarentena, es necesario evaluar el riesgo de su introducción a través de la importación de semilla de pastos, especialmente de países suramericanos.

En vista de lo anterior llevamos a cabo el presente estudio con el objetivo de determinar la situación fitosanitaria de C. benghalensis, determinar la presencia-ausencia de dicha especie en la región sur central y sur de Nicaragua y en caso de ausencia, realizar un análisis de riesgo de introducción de la especie a través de semilla de pastos.

\section{MATERIALES Y MÉTODOS}

El presente estudio es de naturaleza no experimental, de corte transversal. Se llevó a cabo en el periodo comprendido entre el 15 de marzo y el 30 de marzo del año 2012. Para la determinación de la especie Commelina benghalensis, se realizaron giras de reconocimiento en fincas ganaderas en la región central y Atlántico sur (departamentos de Boaco, Chontales, Región Atlántico Sur) y la región sur de Nicaragua (La Virgen y Cárdenas, departamento de Rivas).

Las fincas fueron seleccionadas tomando como criterio el que hayan sido abastecidas de semillas de pastos de parte de los proveedores de servicios agropecuarios en diferentes partes del país. Las fincas seleccionadas se muestran en la tabla 1. 
Tabla 1. Fincas muestreas durante el estudio de prospección de la especie $C$. bengalensis en el periodo comprendido entre el 15 y el 30 de marzo del 2012

\begin{tabular}{llll}
\hline Municipio & Comarca & Nombre del productor & Contacto \\
\hline Nueva Guinea & El Níspero & Concepción Alvarado & Jairo Prado \\
Nueva Guinea & El Níspero & Cesar Alvarado & Jairo Prado \\
Nueva Guinea & Los Pintos & Jairo Prado & Jairo Prado \\
Nueva Guinea & Yolaina & Daniel Prado & Jairo Prado \\
Villa Sandino & Muhan & Orlando Sotelo & Orlando Sotelo \\
Santo Tomás & Las Mesas & Alex Miranda & Orlando Sotelo \\
Santo Tomás & Las Mesas & Wilber Salas & Orlando Sotelo \\
Juigalpa & Batavia & Ignacio Duarte & Juan Carlos Bravo \\
Juigalpa & El Naranjal & Guillermo Duarte & Juan Carlos Bravo \\
Camoapa & Las Lajas & Wilfredo Aragón & Wilfredo Aragón \\
Camoapa & Las Lajas & Enrique Diaz & Wilfredo Aragón \\
Camoapa & Boaco Viejo & Obdulio Sándigo & Wilfredo Aragón \\
Cárdenas & Tirulí & Guillermo Bonnerman & Ramón Guillén \\
Rivas & La Virgen & Carlos Cisnados & Ramón Guillén \\
\hline
\end{tabular}

En cada una de las fincas se estableció un muestreo al azar sistemático. Las fincas fueron recorridas en diagonal, realizando el muestreo (determinación de las especies) de forma visual cada 50 metros. En sitios donde se determinaban especies que se presumía podría ser la especie buscada se recolectaban muestras de las plantas, se corroboraban las características morfológicas de las mismas para luego cotejarlas y aceptar o descartar la presencia de la especie. Las plantas fueron colectadas y trasladadas al campus de la Universidad Nacional Agraria para su determinación.

Posteriormente, en vista de la ausencia de la especie en los campos muestreados, en trabajo de gabinete se realizó una revisión documental con el propósito de hacer una evaluación del riesgo de introducción de $C$. benghalensis a través de la importación de semilla de pastos. Las actividades consistieron en recopilación bibliográfica y consulta a especialistas.

\section{RESULTADOS Y DISCUSIÓN}

Presencia-ausencia de la especie en las áreas muestreadas. No se detectó la presencia de las especie $C$. benghalensis en ninguno de los sitios muestreados. En cinco de las fincas muestreadas se observó la presencia de la especie $C$. erecta, y en seis la presencia de $C$. diffusa. Las especies $C$. erecta y $C$. diffusa fueron colectada, se le determinaron sus características morfológicas y se cotejaron con las características de la especie en estudio. En cada uno de los casos se descartó la presencia de $C$. benghalensis en vista de que no coincidían con las características propias de la especie.

A simple vista las especies del genero Commelina son muy similares, sin embargo existen algunas características de C. benghalensis muy particulares de la especie que facilita descartar la presencia de la especie. Entre ellas se mencionan: la reproducción es sexual, mediante tallos floríferos aéreos, subaéreos y subterráneos que producen semillas viables, pero también es capaz de enraizar desde nodos y propagarse por esquejes de tallos cortados.
Dentro de las especies, se presentan la Commelina diffusa, C. erecta, Murdannia nudiflora y $C$. benghalensis, esta última, reportada en 25 cultivos en 28 diferentes países.

Información del MAGFOR indica la detección de semillas de $C$. benghalensis en semilla de pastos importada desde Brasil. En este país la especie en mención es reportada en diversas cultivos como café, soja, maíz, citrus, cana-de-azúcar, arroz, pasturas, frutales, hortalizas y ornamentales (Correia, 2008), sin embargo, a pesar de la incertidumbre sobre la introducción de la especie o no en Nicaragua, la especie no ha sido determinada en campo.

Commelina benghalensis es una planta de porte bajo más pequeña que las especies gramíneas que se utilizan para alimentar el ganado, su capacidad de competencia es reducida comparada con los pastos. Estas especies sobrepasan a la Commelina lo cual la excluye del sistema, lo que pudiera haber impedido su establecimiento en las condiciones que prevalecen en las pasturas nicaragüenses.

Especies de Commelinas determinadas en campo. A través de los muestreos fue posible determinar dos especies de commelinas, $C$. erecta y $C$. diffusa. Las especies en mención fueron determinadas en el 70 por ciento de las fincas muestreadas, siempre en condiciones de alta humedad, en la proximidad a los afluentes y en condiciones de sombra. A simple vista, las especies en mención poseen características morfológicas que se asemejan a $C$. benghalensis, sin embargo, existen algunas características que permiten discriminar entre ellas.

Commelina diffusa, es una planta suculenta perenne y postrada, de tallos ramificados que producen raíces adventicias en los nudos. Las hojas son lanceoladas, con ápice agudo o acuminado redondeado en la base, la vaina es cerrada, delgada, transparente, seca y membranosa. La inflorescencia se presenta en simas axilares, el pedúnculo es de $1.5 \mathrm{~cm}$ de largo, presenta racimos de 1-3 flores con tres pétalos azules siendo los dos superiores más largos que el inferior, el fruto es una capsula normalmente con cinco semillas de color negro y reticuladas. Se propaga vegetativamente (Alemán et al., 2012). En cambio C. erecta, es también de amplia distribución en el territorio nicaragüense, es una maleza perenne, herbácea, con hábito de crecimiento erecto $\mathrm{o}$ postrado. Se multiplica tanto de forma sexual como asexual. Los tallos son verdes, carnosos y cilíndricos. Las hojas son lanceoladas y sus vainas cubren yemas capaces de producir ramificaciones. Las inflorescencias están protegidas por una espata verde y las flores presentan dos pétalos de color azul o blanco. Los frutos son cápsulas con tres semillas, dos arriñonadas y la tercera ovoide (Nisensohn y Tuesca, 2001). 
En cambio $C$. benghalensis posee una serie de características que la diferencian marcadamente de las especies determinadas. Cristóbal et al., (2008), la describe como una hierba pequeña de hasta $30 \mathrm{~cm}$ de altura, rastrera, formando grandes matas que cubren importantes extensiones (3-4 metros). Se diferencia de las especies descritas anteriormente, en primer lugar en el ancho de la hoja, C. benghalensis posee hojas lanceoladas, sin embargo las hojas son más anchas al compararlas con las otras especies de Commelinas. También se diferencia de las anteriores, en que produce dos clases de frutos: unos que maduran al aire y otros que lo hacen bajo tierra (anficárpica). Las raíces son fasciculadas y entre ellas se observan los tallos subterráneos de color verdoso claro; estos tallos llevan flores cleistógamas de color blanquecino. Una tercera característica que permite discriminar esta especie es la presencia de tricomas de color rojizo en la base de las vainas, característica ausente en las otras especies.

Evaluación del riesgo de introducción de Commelina bengalensis. De la revisión documental efectuada se desprende que las autoridades fitosanitaria nicaragüenses, deberán asegurarse que todas las medidas sanitarias y fitosanitarias que apliquen se basen en estudios de análisis de riesgo. Lo anterior implica contar con talentos humanos dedicados a esa actividad, e infraestructura y recursos materiales que permita efectuar ese tipo de estudios.

Lo anterior es fundamental si se quiere sustentar científica y técnicamente la aplicación de medidas sanitarias y fitosanitarias de importación, de igual forma tener el soporte necesario para declarar materiales importados libres de semillas de malezas, así como zonas libres o de baja prevalencia de una determinada especie nociva.

Se requiere, además, contar con las capacidades necesarias para analizar los estudios de evaluación de riesgo de otros países, el reconocimiento de zonas libres o de baja prevalencia de malezas; pero también para argumentar acerca de medidas de importación impuestas por otros países que obstaculicen operaciones de comercio agropecuario.

FAO (2005) define una serie de criterios que permiten evaluar el riesgo de introducción de especies nocivas. La etapa I, que comprende una exhaustiva revisión bibliográfica que incluye particularidades propias de la especie, como nombres comunes y vernáculos, hábitats y hospederos, distribución geográfica, biología y comportamiento, diseminación, e importancia económica y formas de control, etc., la etapa II que comprende la evaluación del riesgo e introducción y una etapa III que incluye el manejo del riesgo. En este estudio se ha considerado la etapa II y III, evaluación y manejo del riesgo.

La etapa II o evaluación del riesgo es el proceso en el cual se hacen juicios sobre la tolerancia del riesgo en base al análisis de riesgo y tomando en consideración factores socioeconómicos, legales y aspectos ambientales (AS/NZS 2004).
Criterios geográficos $\mathbf{y}$ regulatorios. La especie $C$. benghalensis se clasifica de importancia cuarentenaria (A1) para Nicaragua (Vanegas, 2001). La especie aún no ha sido detectada ni como arvenses y/o como ruderal.

\section{Criterio de importancia económica}

Potencial de entrada. Con la información obtenida a la fecha, la probabilidad de que la maleza sea introducida o haya sido introducida en una importación de semilla de pasto se considera alta debido a que:

- Las detecciones de semilla de $C$. benghalensis reportadas en el pasado de parte de técnicos de la Dirección de Sanidad Vegetal y Semillas, DGPSA-MAGFOR en cargamentos de semillas de pastos importada.

- El tamaño pequeño de las semillas de la maleza y a la posibilidad de no poder ser detectada por medio de examen visual en una inspección rutinaria, puede resultar en su introducción al país.

- La maleza puede sobrevivir fácilmente en las condiciones del transporte debido a su capacidad de dormancia.

La maleza $C$. benghalensis se considera de importancia cuarentenaria en Nicaragua, siendo su potencial de entrada alto a través de la importación de semilla de pastos de países como Brasil donde está presente la maleza.

Potencial de establecimiento. Al tomar en cuenta condiciones climáticas óptimas para su desarrollo, el potencial de establecimiento de la especie $C$. benghalensis se considera alto. La especie tiene posibilidades de sobrevivir y multiplicarse una vez introducida en consideración a los siguientes aspectos:

- Existen cultivos propios de nuestro país, en los cuales se le ha encontrado compitiendo en otras latitudes, i.e., piña (Murillo, 2012), café, tomate, soya, maíz, entro otros (Correia, 2008).

- En nuestro país existen condiciones climáticas óptimas para su desarrollo. El acondicionamiento y germinación de las semillas de la maleza se ven favorecidos por altas temperatura, siendo la óptima de 30 a $35^{\circ} \mathrm{C}$, sin embargo puede desarrollarse en un amplio rango de temperaturas, más allá de $22^{\circ} \mathrm{C}$.

A pesar de lo expuesto, hay consideraciones que pueden derivarse del historial de importación de semillas de pastos en Nicaragua. A pesar de las múltiples detecciones de semillas de $C$. benchalensis en embarques de semillas de pastos procedentes de Brasil, los muestreos de campo no la ubican creciendo junto a la vegetación predominantes en Nicaragua. Lo anterior se sustenta en estudios de prospección que han sido realizados en el occidente, centro y sur de Nicaragua en los últimos cinco años (Datos no publicados).

La tipología de establecimiento de los pastos, los cuales hacen cobertura total del suelo dificulta el establecimiento de la especie, la cual necesita espacio para su establecimiento, y posee poca capacidad de competencia 
con cultivos que la superan en altura. La $C$. benghalensis es una maleza no detectada en Nicaragua, con poca posibilidad de sobrevivir en pastizales por el manto que estas especies hacen sobre el suelo una vez establecidos.

Potencial de propagación después del establecimiento. Este criterio se considera alto. La especie tiene grandes posibilidades de diseminación después de establecerse debido a que:

- Presenta abundante producción de semilla, pero también cuenta con mecanismos de propagación asexual a través de estolones y por su condición anficárpica (planta que produce dos clases de frutos: unos que maduran al aire y otros que lo hacen bajo tierra).

- Fácil diseminación a través del transporte de material infestado, específicamente a través de granos, semillas y residuos vegetales.

Importancia económica potencial. Se considera alto tomando en cuenta los siguientes aspectos:

- La maleza $C$. benghalensis es una malezas agresiva que compite en zonas subtropicales con cultivos como: café, tomate, soya, maíz, entro otros (Correia, 2008).

- La introducción de esta maleza provocaría daños en cultivos plantados en hileras, especialmente en zonas de trópico húmedo.

- Los productos resultantes de la cosecha en cultivos de importancia económica en los cuales potencialmente puede crecer la maleza, disminuirían la calidad al estar contaminado con la semilla de la maleza.

- Se incrementarían los costos de producción del cultivo al tener que aplicarse nuevos métodos de control.
Etapa III: manejo del riesgo. En base al riesgo que representa la maleza, las opciones para disminuir el riesgo a un nivel adecuado son:

En el caso de la semilla de pastos, continuar con los muestreos que permitan determinar niveles críticos de presencia de semillas y propágalos de C. benghalenisis y definir niveles de aceptación de semillas, debido a que el establecimiento de la especie se ve limitada cuando se trata de cultivos que se establecen de cobertura.

En caso de permitirse la importación de semilla de pastos se hacen las siguientes recomendaciones:

- Al ingresar al país se deberán realizar inspección, muestreo y análisis de laboratorio para determinar la presencia o ausencia de $C$. benghalensis. en los embarques.

- El MAGFOR debe solicitar a los importadores de semillas que los embarques de semilla importadas vengan libres de C. benghalensis.

\section{AGRADECIMIENTOS}

Se agradece a la Comisión Nacional de Importadores de Semillas de Pastos (CONISEPA), quienes asumieron los costos de las visitas de campo. A los ingenieros Ramon Guillen Torrentes y Osmin Somarriba (CONISEPA) y Pio Vallecillo y Wilfredo Nicaragua (Dirección General de Sanidad Agropecuaria y Semillas, DGPSA-MAGFOR) quienes participaron en la exploración.

\section{REFERENCIAS BIBLIOGRÁFICAS}

Alemán, F; Quezada, B; Garmendia, M. 2012. Flora arvense y ruderal del pacifico y centro de Nicaragua, Volumen I. Managua, Nicaragua. Universidad Nacional Agraria. 269 p.

Correia, NM; Durigan, JC; Leite, GJ. 2008. Seletividade da soja transgênica tolerante ao glyphosate e eficácia de controle de Commelina benghalensis com herbicidas aplicados isolados e em misturas. Bragantia 67 (3)

Cristóbal, ME; Lozzia, ME; Páez, V. de los A. 2008. Nuevos aportes al conocimiento de Commelina benghalensis L. (Commelinaceae). Lilloa 45 (1-2): 34-38.

Estrada P, BA. 2011. Medidas sanitarias y fitosanitarias en Nicaragua. Cámara de industria y comercio Italo-Nicaraguense. Managua. Nicaragua. 21 p. Revisado: 14 de septiembre, 2012. http://www.camitanica.org.ni/Publicaciones/MSF.pdf

FAO ( Organización de las Naciones Unidas para la Agricultura y la Alimentación, IT). 2005. Procedimientos para la evaluación de riesgos. Dirección de Producción y Protección Vegetal. Departamento de Agricultura.

Grabiele. M; Honfi, AI; Grabiele, M; Debat, HJ; Daviña JR. 2009. Caracterización morfológica y cromosómica de Commelina benghalensis L. (Commelinaceae) de Argentina. Gayana Bot. 66(1). 18-27.

MAGFOR (Ministerio Agropecuario y Forestal, NI). 2002. Norma Técnica Obligatoria Nicaragüense 11 005-02 para la importación de productos y subproductos de origen vegetal y organismos vivos de uso agrícola. Managua, Nicaragua. 14 p. (en línea). Consultado 15 de oct. 2012. Disponible en: http://www.enriquebolanos.org/gacetas_siglo_XXI/Gaceta\%20No.\%20108\%20miercoles\%2011\%20 de $\% 20$ junio $\% 20 \mathrm{de} \% 202003$.pdf

Murillo, RM. Guía de identificación y Manejo integrado de plagas y enfermedades en Piña. (en línea). Consultado 12 de sep. 2012. Ministerio de ambiente, energía y telecomunicaciones. Costa Rica. Disponible en: http://cep.unep.org/repcar/proyectos-demostrativos/ costa-rica-1/publicaciones-anacol/guia\%20identificacion5.pdf

Vanegas-Narváez, ER. 2001. Análisis de riesgo de plagas para la importación de semilla de maní (Arachyis pintoi L.) de los estados Unidos de Norte América. Tesis de grado. Universidad Nacional Agraria. Managua. Nicaragua. 138 p. 